\title{
Intimal relayering technique: Yet another operation for acute aortic dissection
}

\author{
Eugenio Neri, MD, Enrico Tucci, MD, Giulia Guaccio, MD, and Luigi Muzzi, MD, Siena, Italy
}

\author{
From the Institute of Cardiac Surgery, Siena University Hospital, Siena, Italy. \\ No funding was provided for this work. \\ Disclosures: Authors have nothing to disclose with regard to commercial support \\ Received for publication Jan 3, 2018; revisions received March 1, 2018; accepted for publication March 24, 2018; \\ available ahead of print April 22, 2018. \\ Address for reprints: Eugenio Neri, MD, Azienda Ospedaliera Universitaria Senese, Policlinico "Santa Maria alle \\ Scotte.”Viale M. Bracci, Siena 53100, Italy (E-mail: euxneri@tin.it). \\ J Thorac Cardiovasc Surg 2018;156:969-71 \\ $0022-5223 / \$ 36.00$ \\ Copyright (c) 2018 by The American Association for Thoracic Surgery \\ https://doi.org/10.1016/j.jtcvs.2018.03.100
}

Video clip is available online.

Residual dissection after standard hemiarch replacement in type A acute aortic dissection determines the long-term outcome of patients. Arch replacement in acute dissection is a complex procedure that carries a higher risk than elective arch replacement, mainly because of the fragility of dissected vascular tissues. The intimal relayering technique aims to combine the advantages of arch replacement with the simplicity of anterior hemiarch repair while providing an elephant trunk configuration for future interventions.

\section{TECHNIQUE}

Cardiopulmonary bypass is established between the right atrium and the right axillary artery (Video 1). During cooling the aorta is cross-clamped, cold blood retrograde cardioplegia is administered, and proximal aortic repair is accomplished before the circulatory arrest. The arch is prepared to free both sides of the supra-aortic trunks and beyond the origin of the left subclavian artery. The left laryngeal nerve is carefully mobilized to avoid its being trapped in the suture lines. At a systemic temperature of $25^{\circ} \mathrm{C}$, arch vessels are crossclamped and brachiocephalic perfusion started. Once the hemiarch has been opened, a perfusion cannula is inserted into the left common carotid artery.

The arch is inspected, and adequate resection is carried to provide hemiarch repair (Figure 1, A). If the innominate artery is dilated or severely dissected, it is divided for separate reimplantation. The true-lumen size is assessed; to accomplish this, a clear vision of the arch vessel ostia and of the true lumen is obtained with suction in the distal aorta. Under direct vision, a straight Dacron polyester fabric the proximal aorta.

\section{DISCUSSION}

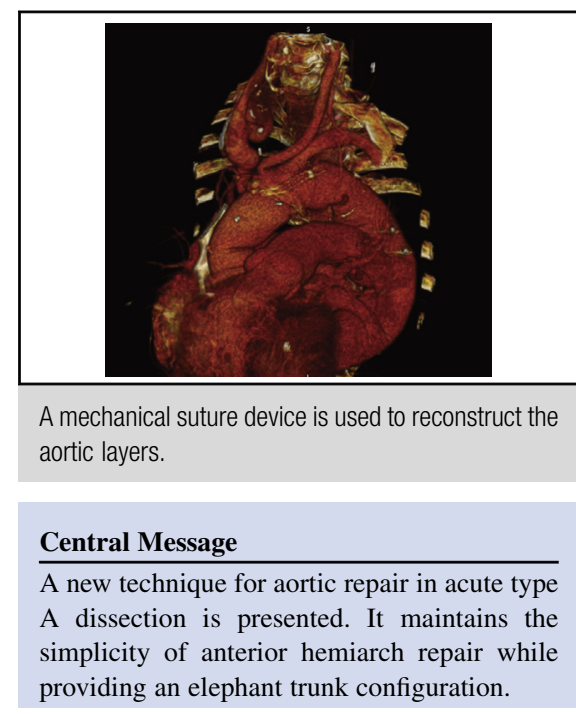

See Editorial Commentary page 972.

(or polytetrafluoroethylene) vascular graft is cautiously inserted into the true lumen (Figure 1,B), with its distal end placed beyond the aortic isthmus, thus creating an elephant trunk. Care must be taken to avoid any intimal damage. A continuous suture (4.0 Prolene with external polytetrafluoroethylene reinforcement; Ethicon, Inc, Somerville, NJ) is performed around the island of supra-aortic trunks, thus securing the graft to the full thickness of the layers of the dissected aorta. To speed up this passage, a bladeless linear stapler (DST Series GIA 60-mm Single Use Knifeless Stapler; Covidien Medtronic, Minneapolis, Minn) has been used in the most recent patients, with optimal quality of suturing (Figure 1,B). The graft is then fenestrated with cautery to provide flow to arch vessels, and the excess of the graft is trimmed along the edge of the aorta (hemiarch; Figure 1, $C$ ). The procedure is then completed in a standard fashion, with the anastomosis of a Dacron polyester fabric graft to replace

In patients undergoing acute type $A$ acute aortic dissection surgery, long-term outcome is strongly dependent on false-lumen patency. ${ }^{1}$ The persistence of a circulating dissection even after repair is attributable to intimal discontinuities along the arch, arch vessels, and descending aorta. Any intervention designed to improve 


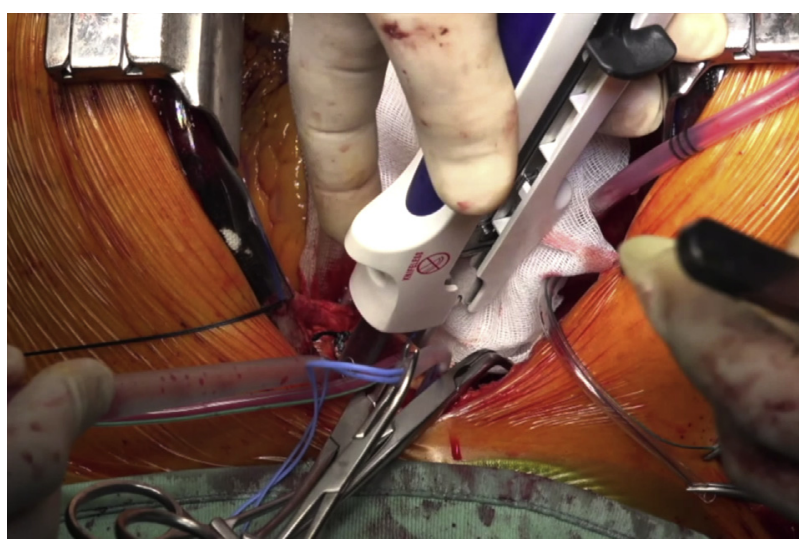

VIDEO 1. Through a median sternotomy and with a deltopectoral incision for axillary artery cannulation, accurate exposure of arch vessels is performed. Care is taken to identify and mobilize the left recurrent laryngeal nerve. Right atrial to axillary cardiopulmonary bypass is established, and moderate hypothermia is obtained. During the circulatory arrest with selective cerebral perfusion, the aorta is opened and the hemiarch is prepared in a standard fashion. A supplemental cannula is inserted into the left common carotid artery $(L C C)$ for bilateral perfusion. A Dacron polyester fabric graft of adequate size is gently inserted into the true lumen; this graft represents the "new intima" and should be "welded" together with the aortic layers. We can use a continuous Prolene suture (Ethicon, Inc, Somerville, NJ) or-as we now prefer-2 bladeless stapler lines on the 2 sides of the supra-aortic trunks. In this way, we separate the island of arch vessels from the rest of the false channel. The suture should therefore be complete all around the vessels. Supplemental stitches (1-2) at the apex of the suture line may be required. To complete the phase of the operation relative to aortic arch repair, the excess of the graft is cut away, and a fenestration, in correspondence with the aortic vessel ostia, is made. The procedure is completed in the usual manner, with a hemiarch replacement. In the illustrated case, the innominate artery (IA) was reimplanted separately. The proximal anastomosis is routinely carried out with a polytetrafluoroethylene sandwich. The control computed tomographic scan demonstrating the stapler lines is shown. The survival curves of the 9 patients treated with this technique and the timeline of the imaging controls are presented. Reinf, Reinforcement; $R C C A$, right common carotid artery; $R S C A$, right subclavian artery; $L C C A$, left common carotid artery; $L S C A$, left subclavian artery; $p t s$, patients; $C P B$, cardiopulmonary bypass; $H C A$, hypothermic circulatory arrest; IQR, interquartile range; $F U$, follow-up; SURV, survival; CENS, censored; $C T$, computed tomographic. Video available at: https:// www.jtcvsonline.org/article/S0022-5223(18)30881-X/fulltext.

intimal continuity by reducing intimal entries thus may improve the outcomes of these patients. On the basis of

inserted inside the true lumen under direct vision. B, The graft is secured to the aortic layers at the level of supra-aortic branches either with a continuous suture or with a bladeless linear stapler. C, The graft is then fenestrated with cautery at the arch vessels, and the excess of the graft is trimmed along the edge of the aorta. (Printed with permission from (c) Campbell Medical Illustration.)

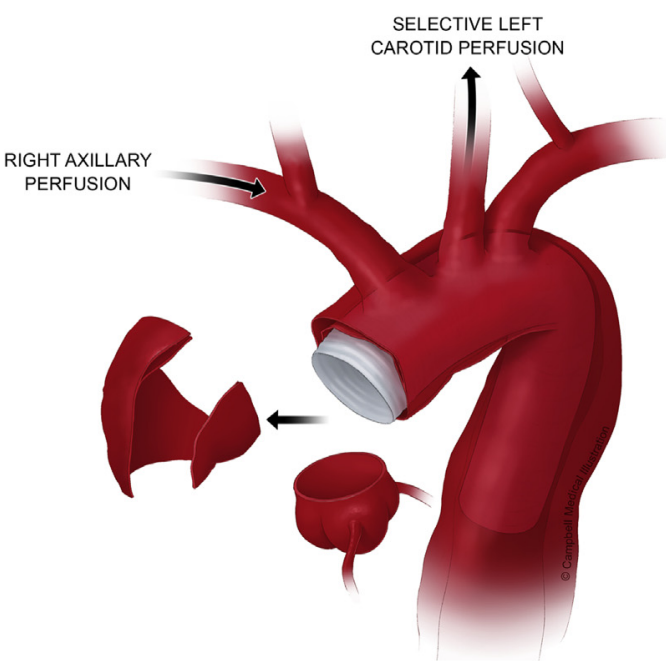

A

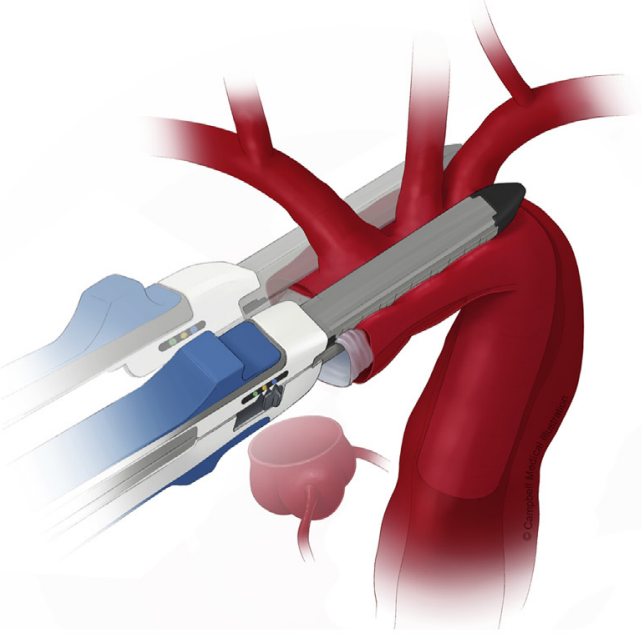

B

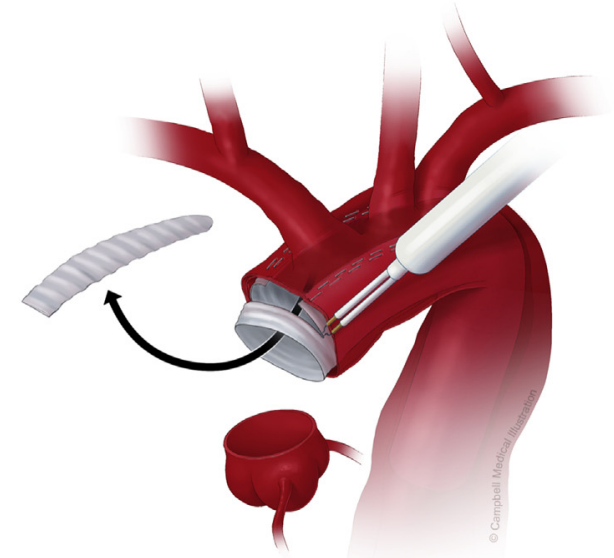

C

FIGURE 1. A, Cardiopulmonary bypass is established between the right atrium and the right axillary artery. The arch is opened, and a perfusion cannula is inserted into the left common carotid artery. A tubular graft is 
these considerations, many authors recommend a total arch replacement on a routine basis in type A acute aortic dissection. $^{2}$

The intimal relayering technique combines the advantages of arch replacement and elephant trunk technique with the simplicity of a standard hemiarch repair. The procedure can be regarded as an adjunct to current hemiarch replacement and as a simplification of the island (en bloc) repair. ${ }^{3}$ The goals are threefold: (1) to reinforce the intima at the arch level with a prosthetic layer; (2) to avoid flow between the false lumen around the arch vessels and that of the distal aorta; and (3) to provide an elephant trunk configuration for further possible interventions in the distal aorta.

Starting in August of 2016, 9 patients were treated with this technique. One patient died on postoperative day 5; at necropsy, the aortic repair was regular. Follow-up is complete for the other 8 patients. In all cases, the procedure effectively promoted channel thrombosis in the upper thoracic aorta, and in no case was an aneurysmal progression of the false channel observed. The use of a bladeless stapler reduced circulatory arrest time while providing an excellent suture line. Median cardiopulmonary bypass time was 122 minutes (interquartile range, 106-135 minutes), hypothermic circulatory arrest time was 34 minutes (interquartile range, 28-37 minutes), and relayering time was 17 minutes (interquartile range, 15-20 minutes). The manual (nonstapled) technique in 2 cases required 27 and 28 minutes. The use of mechanical suturing devices for vessel anastomosis is currently off label.

\section{References}

1. Fattouch K, Sampognaro R, Navarra E, Caruso M, Pisano C, Coppola G, et al Long-term results after repair of type A acute aortic dissection according to false lumen patency. Ann Thorac Surg. 2009;88:1244-50.

2. Shrestha M, Fleissner F, Ius F, Koigeldiyev N, Kaufeld T, Beckmann E, et al Total aortic arch replacement with frozen elephant trunk in acute type A aortic dissections: are we pushing the limits too far?† Eur J Cardiothorac Surg. 2014; 47:361-6.

3. Coselli JS, LeMaire SA, Walkes JC. Surgery for acute type A dissection. Oper Tech Thorac Cardiovasc Surg. 1999;4:13-32. 